\title{
A retrospective cohort study of T3 versus T4 thoracoscopic sympathectomy for primary palmar hyperhidrosis and primary palmar hyperhidrosis with axillary and plantar sweating
}

\author{
Hongya Xie ${ }^{1}$, Tao $\mathrm{Lu}^{2}$, Yimeng Zhu ${ }^{1}$, Donglin Zhu ${ }^{1}$, Tengteng $\mathrm{Wei}^{1}$, Guangda Yuan ${ }^{1}$, Yong Yang ${ }^{1}$, Xiaoqiang Liu ${ }^{1}$ \\ ${ }^{1}$ Department of Thoracic Surgery, The Affiliated Suzhou Hospital of Nanjing Medical University, Suzhou City, Jiangsu Province, China \\ ${ }^{2}$ Department of Thoracic Surgery, Zhongshan Hospital, Fudan University, Shanghai, China
}

Videosurgery Miniinv 2020; 15 (3): 488-495

DOI: https://doi.org/10.5114/wiitm.2019.89656

\begin{abstract}
Introduction: Thoracoscopic sympathectomy (TS) has been proven to be a safe and effective treatment for primary palmar hyperhidrosis (PH). However, the complications include compensatory hyperhidrosis $(\mathrm{CH})$, and over-dry hands may occur in some patients after TS.

Aim: To compare the therapeutic effect of T3 and T4 TS on primary PH and primary PH with axillary and plantar sweating.

Material and methods: We retrospectively analyzed 100 patients with PH who had undergone T3 (group A, $n=49$ ) or T4 (group B, $n=51$ ) TS in our department, with at least 1 year of postoperative follow-up.

Results: At discharge, no major complications or deaths occurred in either group. The condition of sweaty hands was fully improved in 44 of 49 patients in group $A$ and all patients in group $B$, with a significant difference $(p=$ 0.031). After 12 months of follow-up, 18 (36.7\%) patients in group $A$ and 4 (7.8\%) patients in group $B$ developed $C H$, $16(48.5 \%)$ patients in group $A$ and 24 (77.4\%) patients in group $B$ had improved axillary sweating, with a significant difference $(p<0.05)$. The satisfaction rate of group $B$ was significantly higher than that of group $A(p<0.01)$.

Conclusions: Both T3 and T4 TS were safe and effective treatments for PH patients, but the incidence of CH in T4 TS was lower than that in T3 TS. T3 TS may be more suitable for patients with severe PH, while T4 TS had a better therapeutic effect on PH patients with axillary sweating.
\end{abstract}

Key words: palmar hyperhidrosis, thoracoscopic sympathectomy, compensatory hyperhidrosis, axillary sweating, plantar sweating.

\section{Introduction}

Hyperhidrosis is a very embarrassing and distressing condition that affects various body areas, mostly the axillae, soles of the feet, and palms of the hands. It does not threaten health but affects social activities, leading to social withdrawal and even depression. Moreover, because almost everyone now uses smartphones and tablets, palmar hyperhidrosis $(\mathrm{PH})$ has an increasing impact on peo- ple's daily lives. Recently, with the development of minimally invasive thoracoscopic techniques, bilateral thoracoscopic sympathectomy (TS) has been proven to be a safe and effective treatment for $\mathrm{PH}$. However, the complications include compensatory hyperhidrosis $(\mathrm{CH})$, and over-dry hands may occur in some patients after T2 or T2 +3 TS [1, 2]. In addition, some patients with $\mathrm{PH}$ are accompanied by axillary sweat or plantar sweat, or even both. 
To the best of our knowledge, the effect of TS in the treatment of $\mathrm{PH}$ with axillary sweat or plantar sweat is rarely reported in the literature. Therefore, we retrospectively analyzed the patients with $\mathrm{PH}$ who had undergone T4 or T3 TS in our department with at least 1 year of postoperative follow-up, to determine which approach was more effective for $\mathrm{PH}$, axillary sweat and plantar sweat, and which approach had a lower incidence of $\mathrm{CH}$ and other complications.

\section{Aim}

To compare the therapeutic effect and complications of T3 and T4 TS on primary PH, and whether TS is an appropriate treatment for these patients with axillary and plantar sweating.

\section{Material and methods}

\section{Ethics statement}

The study was conducted in accordance with the principles of the Declaration of Helsinki, and the study protocol was approved by the ethics committee of our hospital (register number: 2018(009). All patients signed informed consent and were informed that their data would be used for study purposes.

\section{Patient inclusion and exclusion}

The data were collected from the primary $\mathrm{PH}$ patients who underwent T3 or T4 TS in our department between August 2015 and February 2018. The inclusion criteria were: (a) hand sweat was serious, having become a burden to the professional and personal life; and (b) the course of disease was long, and the effect of drug treatment was poor. Individuals with hyperhidrosis caused by metabolic diseases, tuberculosis, and central diseases were excluded. The degree of palmar hyperhidrosis was classified into three levels as reported by Lai et al. as follows [3]: mild, palms were frequently moist; moderate, palmar sweat was profuse enough to drench a handkerchief; and severe, palmar sweat was spontaneously dripping similar to a faucet leak when the patient made a fist. There were 100 patients with $\mathrm{PH}$ included in this study; 49 patients in group A underwent T3 TS and 51 patients in group B underwent T4 TS only with at least 1 year of postoperative follow-up.

\section{Surgery}

All patients underwent video-assisted single-port thoracoscopic T3/T4 sympathectomy after general anesthesia with double-lumen endotracheal intubation performed by the same experienced surgeons. The patients were given a half-seated position of $30^{\circ}$ to $45^{\circ}$ and abduction of both arms of $90^{\circ}$. Blood pressure, heart rate, and oxygen saturation were monitored during the operation. The temperature of the palm was measured before and after the operation by placing a thermometer in the palm. A $1.5 \mathrm{~cm}$ incision was made in the front line of the third intercostal axilla and the trocar was implanted. The operation was performed by placing a $5 \mathrm{~mm}$ high definition camera for thoracoscopy and an electrocoagulation hook in the same hole. All patient operations followed the right-to-left sequence. The sympathetic nerve chain was free near the spine at the $3 / 4$ rib. Titanium clamps were applied to the upper and lower edges of the $3 / 4$ rib to clamp the fibers of the sympathetic nerve chain. Tissue clamps were used to cut off the T3/T4 sympathetic nerve chain and burn the T3/T4 sympathetic nerve chain, Kuntz bundle, and communicating branches. The temperature rise of the ipsilateral palm was observed during the operation; a more than $1.5^{\circ} \mathrm{C}$ higher temperature than that before operation was judged to be effective. After the operation, the incision was sutured first, and the thoracic drainage tube was temporarily placed. The drainage tube was quickly removed and the incision was sutured after exhausting the pleural cavity. The operation of the other side was performed in the same way after one side of the operation.

\section{Observation indices}

The sex, age, degree of palmar hyperhidrosis, axillary sweat, plantar sweat, occupation, and body mass index (BMI) of the patients were evaluated before the operation (Table I). The occupations of patients were divided into manual workers and mental workers. The duration of the operation, length of hospital stays, effect of the operation, and complications were observed during the perioperative period (Table II). The complications included: over-dry hands, compensatory sweating, pneumothorax, Horner syndrome, intercostal neuralgia, hemothorax, and surgical site infections. The side effects, including over-dry hands and compensatory 
Table I. Baseline characteristics of patients before operation

\begin{tabular}{|c|c|c|c|}
\hline Parameter & Group A $(n=49)$ & Group B $(n=51)$ & $P$-value \\
\hline Age [years] & $21.63 \pm 4.05$ & $21.08 \pm 3.83$ & 0.483 \\
\hline Sex: & & & 0.250 \\
\hline Male & $36(73.5)$ & $32(62.7)$ & \\
\hline Female & $13(26.5)$ & $19(37.3)$ & \\
\hline Family history & $22(44.9)$ & $19(37.3)$ & 0.437 \\
\hline Degree of palmar hyperhidrosis: & & & 0.530 \\
\hline Moderate & $20(40.8)$ & $24(47.1)$ & \\
\hline Severe & $29(59.2)$ & $27(52.9)$ & \\
\hline Axillary sweat & $33(67.3)$ & $31(60.8)$ & 0.494 \\
\hline Plantar sweat & $25(51.0)$ & $30(58.8)$ & 0.433 \\
\hline Occupations: & & & 0.076 \\
\hline Manual workers & $5(10.2)$ & $12(23.5)$ & \\
\hline Mental workers & $44(89.8)$ & $39(76.5)$ & \\
\hline BMI $\left[\mathrm{kg} / \mathrm{m}^{2}\right]$ : & & & $0.581^{a}$ \\
\hline$<24$ & $46(93.9)$ & $50(98.0)$ & \\
\hline$\geq 24$ & $3(6.1)$ & $1(2.0)$ & \\
\hline
\end{tabular}

aFisher's exact test was used instead, as there were expected cell frequencies < 5. Data are mean \pm SD or $n(\%)$.

Table II. Postoperative complications and early surgical effect

\begin{tabular}{|lccc|}
\hline Parameter & Group A $(n=49)$ & Group B $(n=51)$ & $P$-value \\
\hline Duration of operation [min] & $27.53 \pm 1.55$ & $28.63 \pm 1.34$ & 0.237 \\
\hline Duration of hospital stay [days] & $2.12 \pm 0.82$ & $2.32 \pm 1.05$ & 0.325 \\
\hline \begin{tabular}{l} 
Early effect of surgery: \\
\hline Dry hands
\end{tabular} & $44(89.8)$ & $38(77.6)$ & 0.101 \\
\hline Mild moist hands & $5(10.2)$ & $6(11.8)$ & 0.083 \\
\hline Wet hands & $0(0)$ & $5(9.8)$ & $0.031^{a}$ \\
\hline Improvement of axillary sweating & $17(51.5)$ & $24(77.4)$ & 0.031 \\
\hline Improvement of plantar sweating & $10(40.0)$ & $13(43.3)$ & 0.765 \\
\hline Pneumothorax & $4(8.2)$ & $3(5.9)$ & $0.477^{\mathrm{a}}$ \\
\hline Horner syndrome & $0(0)$ & $0(0)$ & - \\
\hline Intercostal neuralgia & $2(4.1)$ & $1(2.0)$ & $0.485^{\mathrm{a}}$ \\
\hline Hemothorax & $0(0)$ & $0(0)$ & - \\
\hline Surgical site infection & $0(0)$ & $0(0)$ & - \\
\hline
\end{tabular}

${ }^{a}$ Fisher's exact test was used instead, as there were expected cell frequencies $<5$. Data are mean \pm SD or $n(\%)$.

hyperhidrosis $(\mathrm{CH})$, the status of wet hands, and the and 12 months in the course of the follow-up (Taimprovement of axillary and plantar sweating were ble III). The degree of postoperative $\mathrm{CH}$ was classievaluated at the time points of 1 month, 6 months, fied as follows: mild (undergarments remained dry 
Table III. Results of follow-up

\begin{tabular}{|c|c|c|c|c|c|c|c|c|c|}
\hline \multirow[t]{2}{*}{ Parameter } & \multicolumn{3}{|c|}{ After 1 month of follow-up } & \multicolumn{3}{|c|}{ After 6 months of follow-up } & \multicolumn{3}{|c|}{ After 12 months of follow-up } \\
\hline & Group A & Group B & $P$-value & Group A & Group B & $P$-value & Group A & Group B & $P$-value \\
\hline \multicolumn{10}{|l|}{$\begin{array}{l}\text { Compensatory } \\
\text { hyperhidrosis: }\end{array}$} \\
\hline Mild & $12(24.5)$ & $4(7.8)$ & $0.030^{a}$ & $12(24.5)$ & $4(7.8)$ & 0.030 & $12(24.5)$ & $3(5.9)$ & 0.012 \\
\hline Moderate & $4(8.2)$ & $2(3.9)$ & $0.432^{a}$ & $3(6.1)$ & $1(2.0)$ & $0.357^{a}$ & $3(6.1)$ & $1(2.0)$ & $0.357^{a}$ \\
\hline Severe & $5(10.2)$ & $0(0)$ & $0.025^{a}$ & $3(6.1)$ & $0(0)$ & $0.114^{a}$ & $3(6.1)$ & $0(0)$ & $0.114^{a}$ \\
\hline Total & $21(42.9)$ & $6(11.8)$ & 0.000 & $18(36.7)$ & $5(9.8)$ & 0.001 & $18(36.7)$ & $4(7.8)$ & $0.001^{a}$ \\
\hline Over-dry hands & $7(14.3)$ & $6(11.8)$ & 0.708 & $6(12.2)$ & $6(11.8)$ & 0.941 & $6(12.2)$ & $6(11.8)$ & 0.941 \\
\hline Wet hand & $0(0)$ & $5(9.8)$ & $0.031^{\mathrm{a}}$ & $1(2.0)$ & $7(13.7)$ & $0.034^{a}$ & $1(2.0)$ & $7(13.7)$ & $0.034^{a}$ \\
\hline $\begin{array}{l}\text { Improvement of } \\
\text { axillary sweating }\end{array}$ & $17(51.5)$ & $24(77.4)$ & 0.031 & $17(51.5)$ & $24(77.4)$ & 0.031 & $16(48.5)$ & $24(77.4)$ & 0.017 \\
\hline $\begin{array}{l}\text { Improvement of } \\
\text { plantar sweating }\end{array}$ & $10(40.0)$ & $13(43.3)$ & 0.765 & $13(52.0)$ & $17(56.7)$ & 0.729 & $13(52.0)$ & $16(53.3)$ & 0.921 \\
\hline \multicolumn{10}{|l|}{ Patient satisfaction: } \\
\hline Very satisfied & $23(46.9)$ & $44(86.3)$ & 0.000 & $21(42.9)$ & $43(84.3)$ & 0.000 & $21(42.9)$ & $42(82.4)$ & 0.000 \\
\hline Satisfied & $4(8.2)$ & $2(3.9)$ & $0.637^{a}$ & $2(4.1)$ & $2(3.9)$ & $0.676^{a}$ & $3(6.1)$ & $3(5.9)$ & $0.642^{a}$ \\
\hline Partially satisfied & $22(44.9)$ & $5(9.8)$ & 0.000 & $26(53.1)$ & $6(11.8)$ & 0.000 & $26(53.1)$ & $7(13.7)$ & 0.000 \\
\hline
\end{tabular}

aFisher's exact test was used instead, as there were expected cell frequencies < 5. Data are mean \pm SD or $n(\%)$.

despite heavy sweating); moderate (undergarments were sometimes soaked with sweat but were tolerable); and severe (undergarments were soaked with excessive sweat and became intolerable, requiring several changes a day). Data on the patients' postoperative status were collected by regular visits in the outpatient clinic and by direct telephone contact.

\section{Statistical analysis}

Continuous data were expressed as the mean \pm standard deviation (SD). Significance of differences between the two groups was determined by the $\chi^{2}$ test or Student's $t$-test. Fisher's exact test was used instead if there were expected cell frequencies less than 5. All statistical analyses were carried out using SPSS Statistics, version 24.0 (IBM, New York, NY, USA), and $p<0.05$ was considered significant.

\section{Results}

Baseline characteristic data before the operations are presented in Table I. Both groups were mainly young males. There was no significant difference in age between the two groups (21.63 \pm 4.05 vs. $21.08 \pm 3.83$ years, $p=0.483)$. No significant dif- ference was observed in terms of $\operatorname{sex}(p=0.250)$, family history $(p=0.437)$, degree of palmar hyperhidrosis $(p=0.530)$, axillary sweat $(p=0.494)$, plantar sweat $(p=0.433)$, occupation $(p=0.076)$, or BMI $(p=0.581)$.

The data concerning postoperative complications and early surgical effects are presented in Table II. No major complications or deaths occurred in either group, and no patient developed Horner syndrome, hemothorax, or a surgical site infection in either group. Only 2 (4.1\%) patients in group A and $6(2.0 \%)$ patients in group B had intercostal neuralgia, and there was no significant difference between the two groups $(p=0.485)$. Pneumothorax was detected in $4(8.2 \%)$ patients in group A and 3 (5.9\%) patients in group $B$, with no significant difference between the groups ( $p=0.477)$. No significant difference was observed in duration of the operation $(27.53 \pm 1.55 \mathrm{~min}$ vs. $28.63 \pm 1.34 \mathrm{~min}, p=0.237)$ or duration of the hospital stay (2.12 \pm 0.82 days vs. $2.32 \pm 1.05$ days, $p=0.325$ ) between the two groups.

At discharge, the condition of sweaty hands was fully controlled in 44 of 49 patients in group $A$ and all patients in group $B$, with a significant difference between the two groups $(p=0.031)$. In addition, in all 
$5(9.8 \%)$ patients without improvement, their sweaty hands had severe $\mathrm{PH}$ before surgery. Seventeen (51.5\%) patients from group A and 24 (77.4\%) patients from group $B$ had improved axillary sweating, with significant differences between the two groups $(p=0.031)$. There were $10(40.0 \%)$ patients in group $A$ and 13 (43.3\%) patients in group B with improvements of plantar sweating, and there was no significant difference between the two groups ( $p=0.765)$.

The results of follow-up examinations are presented in Table III. With the prolongation of follow-up time, $\mathrm{CH}$ patients in both groups decreased gradually. The number of patients with $\mathrm{CH}$ were 21 $(42.9 \%)$ in group A and $6(11.8 \%)$ in group B (after 1 month of follow-up), 18 (36.7\%) in group $A$ and $5(9.8 \%)$ in group B (after 6 months of follow-up), 18 (36.7\%) in group A and 4 (7.8\%) in group B (after 12 months of follow-up), with significant differences between the two groups $(p<0.01)$.

After 1 month of follow-up, the condition of $5(9.8 \%)$ patients with wet hands in group B at the time of discharge was not improved, while there was no patient with wet hands in group $A$, with a significant difference between the two groups ( $p=0.031$ ). At 12 months of follow-up, 1 patient in group A had a recurrence of $\mathrm{PH}$, and 2 patients in group $\mathrm{B}$ had a recurrence of $\mathrm{PH}$. Because there was 1 (2.0\%) patient in group $A$ and 7 (13.7\%) patients in group $B$ having wet hands, there was a significant difference between the two groups ( $p=0.034)$.

After 1 and 6 months of follow-up, there was no change in the number of patients whose axillary sweat improved between the two groups as compared with that at the time of discharge. There was 1 patient in group A having a recurrence of axillary sweat after a 12-month follow-up, while patients in group $B$ showed no change, with a significant difference between the two groups ( $p=0.017$ ).

There were 10 patients in group $A$ and $13 \mathrm{pa}$ tients in group B whose plantar sweating improved when they were discharged after 1 month of follow-up. The number of patients whose plantar sweating improved was 13 (52.0\%) in group A and $17(56.7 \%)$ in group B (after 6 months of follow-up) and 13 (52.0\%) in group $A$ and 16 (53.3.7\%) in group $B$ (after 12 months of follow-up), with no significant difference between the two groups $(p>0.05)$.

At the first month of follow-up, there were 23 (46.9\%) patients in group A and 44 (86.3\%) patients in group $B$ who were very satisfied with TS, with a significant difference between the two groups ( $p<0.001$ ). As the follow-up time increased, the patients' satisfaction decreased slightly. The number of patients who were very satisfied with TS were 21 (42.9\%) in group A and 42 (84.3\%) in group B (after 6 months of follow-up), 21 (42.9\%) in group $A$ and 42 (82.4\%) in group B (after 12 months of follow-up), with a significant difference between the two groups $(p<0.001)$.

\section{Discussion}

At present, TS is the preferred surgical treatment for $\mathrm{PH}$. The procedure used in the treatment of $\mathrm{PH}$ is to cut the thoracic sympathetic nerve chain and block the postganglionic fibers. There are different surgical approaches that were used over time with different outcomes [4]. Conventionally, the main innervation pathway was the T2 ganglion for the hands, and most thoracic sympathectomies were performed at this site $[5,6]$. However, the incidence of $\mathrm{CH}$ is often high after $\mathrm{T} 2$ thoracic sympathectomy, which affects the quality of life of patients [2, 7]. Reisfeld's research has shown that sympathetic nerve chains from low to high continuously had sympathetic nerve fibers added to the upstream fibers; the higher the level of surgical cut-off, the larger the sympathetic range will be, and the $\mathrm{CH}$ will be more severe [8]. According to the anatomical characteristics of the thoracic sympathetic nerve chain, reducing the cut-off plane of the sympathetic nerve chain should be helpful in reducing the scope and degree of surgical sympathetic denervation to make the surgical sympathetic denervation effect more targeted. Turhan et al. compared T2 and T3 sympathectomy and suggested that denervation of the T2 ganglion should be avoided to decrease the incidence of $\mathrm{CH}$ [7]. Since then, T3 and T4 sympathectomy procedures have been widely used for low incidences of complications such as $\mathrm{CH}$ [9-11]. The complications of TS have been previously described, and include intercostal neuralgia, recurrence, $\mathrm{CH}$, Horner syndrome, pneumothorax, hemothorax, and surgical site infection. In our study, there was no Horner syndrome, hemothorax, or surgical site infection. Pneumothorax occurred in $7(7.0 \%)$ patients of the two groups, and all these patients were treated conservatively because the size of the pneumothorax in the chest X-ray was less than $20 \%$. Most intercostal neuralgia is caused by the destruction and compression 
of intercostal nerves during surgery. Thanks to the use of a single hole and a $5 \mathrm{~mm}$ camera, the effect of the operation on the intercostal nerve was minimized. Therefore, in our study, only $3(3.0 \%)$ patients developed mild intercostal neuralgia. This incidence was very low compared with the $15-30 \%$ reported by other surgeons [12, 13]. Moreover, all 3 patients with intercostal neuralgia improved after being treated with oral nonsteroidal anti-inflammatory drugs.

To date, $\mathrm{CH}$ is still the most troublesome complication after TS [14]. It is difficult to precisely estimate the incidence of $\mathrm{CH}$, and reports vary in the literature, from $3 \%$ to $100 \%$ [15]. In a prospective randomized study, $\mathrm{PH}$ was treated by TS at either the T2 or T3 level. Success was $100 \%$ in the T2 group and $97 \%$ in the $\mathrm{T} 3$ group, with the incidence of $\mathrm{CH}$ being $100 \%$ in the $\mathrm{T} 2$ group and $97 \%$ in the $\mathrm{T} 3$ group [16]. Another study compared T2-T4 TS to T3-T4 TS for $\mathrm{PH}$ and did not find any significant difference between the two groups [17]. In our study, there were 18 (36.7\%) patients in group A and $4(7.8 \%)$ patients in group B with $\mathrm{CH}$ after 12 months of follow-up. $\mathrm{CH}$ is believed to be due to the disruption of the reflex arc between sympathetic nerve activity and the hypothalamus, resulting in a disturbance of sweating control. The preservation of the negative afferent tone to the hypothalamus is the key to preventing postoperative $\mathrm{CH}$. This is a convincing theoretical hypothesis for $\mathrm{CH}$, which has been confirmed by additional clinical studies [18, 19]. Dewey et al. reported that limited TS for PH to a single denervation level reduced the incidences of severe $\mathrm{CH}$ [20]. A similar result has been reported by Wolosker et al.; adjusting the denervation levels from $\mathrm{T} 3$ to $\mathrm{T} 4$ reduced the incidences of severe $\mathrm{CH}$ [21]. However, another compensation theory suggests that after elimination of hyperhidrosis in the target area, the body produces an increase in sweating in other skin areas in order to maintain the balance of sweating in the whole body. In other words, the more severe the hand sweating is before the operation, the more the original hand sweating is transferred to other areas after the operation, and the more serious the compensatory sweating is, which is consistent with a previous report [22].

Patients with PH often also have axillary and plantar hyperhidrosis. Sympathectomy is not the preferred treatment for mild axillary hyperhidrosis because of side effects [23]. The treatment of mild axillary hyperhidrosis includes topical aluminium chloride, iontophoresis, injection of botulinum toxin $\mathrm{A}$, oral medication, surgical methods, laser treatment, and microwave thermolysis, with the latter two treatments particularly being accepted by more patients because of their positive effects, less pain, and less trauma [24]. However, for patients with PH and axillary hyperhidrosis, TS may be the best treatment choice. Jeganathan et al. reported that T4-T5 TS for axillary hyperhidrosis had a satisfactory effect [25]. In fact, primary plantar hyperhidrosis usually needs a lumbar sympathectomy instead of TS, which is currently the preferred approach, including that reported in a small case series study [26, 27]. Rieger et al. carried out a case series study of 178 endoscopic resection lumbar sympathectomies in 90 patients with severe plantar hyperhidrosis. After a follow-up of 3-45 months, $97 \%$ of the patients had eliminated their plantar hyperhidrosis [28]. However, studies also reported a loss of sexual function in males after lumbar sympathectomy [29]. In our study, because the main symptom of the patients was $\mathrm{PH}$, axillary sweating and plantar sweating were only subsidiary symptoms. Therefore, we did not perform T4 + 5 TS for axillary hyperhidrosis or lumbar sympathectomy for plantar sweating. Nonetheless, 16 (48.5\%) patients in group A and 24 (77.4\%) patients in group B had improved axillary sweating after 12 months follow-up, with a significant difference compared with group A, which indicated that T4 TS was better than T3 TS for PH patients with axillary sweating. However, there were 13 (52.0\%) patients in group $A$ and $16(53.3 \%)$ patients in group B with improvement of plantar sweating after 12 months of follow-up, and there was no significant difference between the two groups, which indicated that T3 and T4 TS could at least partially improve plantar sweating.

As previously reported, some patients still had wet hands after surgery, which was due to the failure of surgery. Studies have shown that recurrence of PH was probably due to incomplete sympathectomy or the regeneration of sympathetic nerves to the stellate ganglion [30]. Misidentification of the sympathetic chain due to local anatomic variations, localized pleural thickening, or adhesions were also possible causes. In our study, we noted that 5 (9.8\%) patients with wet hands in group $B$ had unsatisfactory surgical effects at discharge, which was due to the failure of the operation. Moreover, 2 (3.9\%) patients had recurrence of PH at the 6-month follow-up, while all patients in group $A$ achieved a satisfactory 
effect at discharge, and only 1 (2.0\%) patient had recurrence after 6 months of follow-up. In addition, all 8 patients with wet hands in both groups had severe $\mathrm{PH}$ before the operation. Therefore, our study suggested that T3 TS may be more effective than T4 TS in patients with severe $\mathrm{PH}$. This result differed somewhat from that previously reported $[9,10,21]$.

To our knowledge, no studies on the mechanism of over-dry hands have been reported, despite the reported incidence of over-dry hands being as high as $35 \%[4,31]$. In our study, over-dry hands were present in $7(14.3 \%)$ patients in group $A$ and $6(11.8 \%)$ patients in group B after 1 month of follow-up, and with increased time, 1 patient in group $A$ had an improved dry hand condition. In addition, compared with $\mathrm{CH}$ and wet hands, over-dry hands had less impact on the daily life of patients, and could be solved by regular use of hand cream. The main factors affecting patients' satisfaction were the effects of surgery and $\mathrm{CH}$. According to previous studies, the patient satisfaction ranged from $35 \%$ to $100 \%$ [32]. In our study, at the first month of follow-up, the satisfaction levels of patients in group A and group B were $46.9 \%$ and $86.3 \%$, respectively. With the increase of follow-up time, patient satisfaction decreased slightly, probably due to the recurrence of $\mathrm{PH}$ and the increase of $\mathrm{CH}$.

\section{Conclusions}

Both T3 and T4 TS are safe and effective treatments for $\mathrm{PH}$ patients, but the incidence of $\mathrm{CH}$ in $\mathrm{T} 4$ TS was lower than that in T3 TS, which is consistent with most previously reported results. However, for patients with severe $\mathrm{PH}, \mathrm{T} 3 \mathrm{TS}$ may have a better effect than T4 TS. Moreover, T4 TS had a better therapeutic effect on $\mathrm{PH}$ patients with axillary sweating. However, both T3 and T4 TS could at least partially improve plantar sweating. The satisfaction rate of T4 TS was significantly higher than that of T3 TS.

\section{Acknowledgments}

Hongya Xie and Tao Lu contributed equally to this work. The authors thank International Science Editing (http://www.internationalscienceediting.com) for editing this manuscript.

\section{Conflict of interest}

The authors declare no conflict of interest.

\section{References}

1. Yazbek G, Wolosker N, Kauffman P, et al. Twenty months of evolution following sympathectomy on patients with palmar hyperhidrosis: sympathectomy at the T3 level is better than at the T2 level. Clinics 2009; 64: 743-9.

2. Baumgartner FJ, Reyes M, Sarkisyan GG, et al. Thoracoscopic sympathicotomy for disabling palmar hyperhidrosis: a prospective randomized comparison between two levels. Ann Thorac Surg 2011; 92: 2015-9.

3. Lai YT, Yang LH, Chio CC, Chen HH. Complications in patients with palmar hyperhidrosis treated with transthoracic endoscopic sympathectomy. Neurosurgery 1997; 41: 110-3.

4. Rodríguez PM, Freixinet IL, Mohamed H, et al. Side effects, complications and outcome of thoracoscopic sympathectomy for palmar and axillary hyperhidrosis in 406 patients. Eur J Cardiothorac Surg 2008; 34: 514-9.

5. Goh PMY, Cheah WK, Costa MD, et al. Needlescopic thoracic sympathectomy: treatment for palmar hyperhidrosis. Ann Thorac Surg 2000; 70: 240-2.

6. Lee DY, Yoon YH, Shin HK, et al. Needle thoracic sympathectomy for essential hyperhidrosis: intermediate-term follow-up. Ann Thorac Surg 2000; 69: 251-3.

7. Turhan K, Cakan A, Cagirici U. Preserving T2 in thoracic sympathicotomy for palmar hyperhidrosis: less tissue trauma, same effectiveness. Thorac Cardiovasc Surg 2011; 59: 353-6.

8. Reisfeld R. Sympathectomy for hyperhidrosis: should we place the clamps at T2-T3 or T3-T4? Clin Auton Res 2006; 16: 384-9.

9. Kim WO, Kil HK, Yoon KB, et al. Influence of T3 or T4 sympathicotomy for palmar hyperhidrosis. Am I Surg 2010; 199: 166-9.

10. Ishy A, de Campos JR, Wolosker N, et al. Objective evaluation of patients with palmar hyperhidrosis submitted to two levels of sympathectomy: T3 and T4. Interact Cardiovasc Thorac Surg 2011; 12: 545-8.

11. Bagheri R, Sharifian Attar A, Haghi SZ, et al. Thoracoscopic sympathicotomy in the treatment of palmar hyperhidrosis. Asian Cardiovasc Thorac Ann 2016; 24: 687-91.

12. Buraschi J. Videothoracoscopic sympathicolysis procedure for primary palmar hyperhidrosis in children and adolescents. Arch Argent Pediatr 2008; 106: 32.

13. Angerpointner TA. Thoracoscopic sympathectomy for palmar hyperhidrosis in children: 21 years of experience at a tertiary care center. J Pediatr Surg 2013; 23: 486-9.

14. Cheng A, Johnsen H, Chang MY. Patient satisfaction after thoracoscopic sympathectomy for palmar hyperhidrosis: do method and level matter? Perm J 2015; 19: 29-31.

15. Cerfolio RJ, de Campos JRM, Bryant AS, et al. The Society of Thoracic Surgeons expert consensus for the surgical treatment of hyperhidrosis. Ann Thorac Surg 2011; 91: 1642-8.

16. Guilherme Y, Nelson W, de Campos JRM, et al. Palmar hyperhidrosis: which is the best level of denervation using video-assisted thoracoscopic sympathectomy: T2 or T3 ganglion? J Vasc Surg 2005; 42: 281-5.

17. Scognamillo F, Serventi F, Attene F, et al. T2-T4 sympathectomy versus T3-T4 sympathicotomy for palmar and axillary hyperhidrosis. Clin Auton Res 2011; 21: 97-102. 
18. Lin CC, Telaranta T. Lin-Telaranta classification: the importance of different procedures for different indications in sympathetic surgery. Ann Chir Gynaecol 2001; 90: 161-6.

19. Thorlacius L, Gyldenløve M, Zachariae C, et al. Distinguishing hyperhidrosis and normal physiological sweat production: new data and review of hyperhidrosis data for 1980-2013. Int J Dermatol 2015; 54: e409-15.

20. Dewey TM, Herbert MA, Hill SL, et al. One-year follow-up after thoracoscopic sympathectomy for hyperhidrosis: outcomes and consequences. Ann Thorac Surg 2006; 81: 1227-32.

21. Wolosker N, Yazbek G, A, De Campos J, et al. Is sympathectomy at T4 level better than at T3 level for treating palmar hyperhidrosis? I Laparoendosc Adv Surg Tech A 2008; 18: 102-6.

22. Min L, Tu Y, Xu L. Follow-up observations of 200 cases of endoscopic thoracic sympathectomy for palmar hyperhidrosis. Chin J Minim Inv Surg 2005.

23. Singh S, Davis H, Wilson P. Axillary hyperhidrosis: a review of the extent of the problem and treatment modalities. Surgeon 2015; 13: 279-85.

24. Hsu TH, Chen YT, Tu YK, et al. A systematic review of microwave-based therapy for axillary hyperhidrosis. I Cosmet Laser Ther 2017; 19: 275-82.

25. Jeganathan $R$, Jordan $S$, Jones $M$, et al. Bilateral thoracoscopic sympathectomy: results and long-term follow-up. Interact Cardiovasc Thorac Surg 2008; 7: 67-70.

26. Marlos C, William K, Luciano Carneiro S, et al. Bilateral retroperitoneoscopic lumbar sympathectomy by unilateral access for plantar hyperhidrosis in women. J Laparoendosc Adv Surg Techn A 2010; 20: 1-6.

27. Nyamekye IK. Current therapeutic options for treating primary hyperhidrosis. Eur J Vasc Endovasc Surg 2004; 27: 571-6.

28. Naumann MK, Hamm H, Lowe NJ. Effect of botulinum toxin type $\mathrm{A}$ on quality of life measures in patients with excessive axillary sweating: a randomized controlled trial. Br I Dermatol 2015; 147: 1218-26

29. Rieger R, Pedevilla S, Pöchlauer S. Endoscopic lumbar sympathectomy for plantar hyperhidrosis. J Vasc Surg 2010; 52: 252-2.

30. Sinha CK, Edward K. Thoracoscopic sympathectomy for palmar hyperhidrosis in children: 21 years of experience at a tertiary care center. J Pediatr Surg 2013; 23: 486-9.

31. Yanguo L, Jie Y, Jun L, et al. Surgical treatment of primary palmar hyperhidrosis: a prospective randomized study comparing T3 and T4 sympathicotomy. Eur J Cardiothor Surg 2009; 35: 398-402.

32. Johannes S, Falk Georges B, Peter A, et al. Endoscopic thoracic sympathectomy for severe hyperhidrosis: impact of restrictive denervation on compensatory sweating. Ann Thorac Surg 2006; 81: 1048-55.

Received: 18.09.2019, accepted: 15.10.2019. 